\title{
Synaptic Vesicle Protein 2 and Vesicular Monoamine Transporter 1 and 2 Are Expressed in Neuroblastoma
}

\author{
Kleopatra Georgantzi ${ }^{1}$ (D) - Apostolos V. Tsolakis ${ }^{2,3,4} \cdot$ Åke Jakobson $^{1} \cdot$ Rolf Christofferson ${ }^{1}$ • Eva Tiensuu Janson ${ }^{4}$. \\ Lars Grimelius ${ }^{5}$
}

Published online: 18 July 2019

(C) The Author(s) 2019

\begin{abstract}
Neuroblastoma (NB), the most common extracranial cancer in childhood, exhibits neuroendocrine (NE) differentiation. Two well-established NE markers, chromogranin A ( $\mathrm{CgA}$ ) and synaptophysin (syn), are used in the histopathological diagnostics. Our aims were to explore if the NE markers synaptic vesicle protein 2 (SV2) and vesicular monoamine transporter 1 (VMAT1) and 2 (VMAT2) also are expressed in human NB and if so, evaluate their usefulness in NB histopathological diagnostics. Tumor specimens from $21 \mathrm{NB}$ patients, before and/or after chemotherapy, were immunostained for CgA, syn, SV2, VMAT1, and VMAT2. Clinical data was extracted from patients' records. SV2 was highly expressed in NB, as was CgA while syn was less frequently expressed compared to the other two. Both VMATs were expressed in several NB, VMAT2 in more cases than VMAT1 and its expression was similar to syn. Chemotherapy did not affect the immunoreactivity in an obvious way. SV2 was highly expressed in NB and can thus be useful marker in NB diagnostics. VMAT1 and VMAT2 were also expressed in NB but similar to syn less reliable as tumor markers.
\end{abstract}

Keywords Neuroblastoma $\cdot$ Neuroendocrine $\cdot$ Immunohistochemistry $\cdot$ Markers

\section{Introduction}

Neuroblastoma (NB) is the most common extracranial tumor in children, originating from cells of the adrenal medulla or the sympathetic nervous system. NB exhibits a unique biological and clinical heterogeneity - some tumors may differentiate or even regress spontaneously, and others progress or relapse despite aggressive, multimodal therapy.

Kleopatra Georgantzi

clary.georgantzi@kbh.uu.se

1 Department of Women's and Children's Health, Uppsala University, Uppsala, Sweden

2 Department of Oncology and Pathology, Karolinska Institute, Stockholm, Sweden

3 Cancer Center Karolinska, CCK, Karolinska University Hospital Solna, Stockholm, Sweden

4 Department of Medical Sciences, Uppsala University, Uppsala, Sweden

5 Department of Immunology, Genetics and Pathology, Uppsala University, Uppsala, Sweden
NB shows neuroendocrine (NE) properties. A NE phenotype can be assessed by the expression of general and specific NE markers. Chromogranin A (CgA), a general NE marker, and the more specific markers vesicular monoamine transporter 1 and 2 (VMAT1 and VMAT2) are associated with large dense core vesicles. Other general markers, e.g., synaptophysin (syn) and synaptic vesicle protein 2 (SV2), correspond to small synaptic-like vesicles [1-5]. $\mathrm{CgA}$ and syn are cornerstones in the histopathological diagnostics of neuroendocrine tumors (NETs) including NB [6]. Another broad spectrum NE marker, INSM1, has been used frequently in the past 4 years for the workup of NETs [7]. This biomarker has been reported to stain NB [8].

SV2s are a family of three membrane proteoglycans (SV2A, B, and C), specifically found in the secretory vesicles of neural and NE cells [9]. SV2A is localized in the pancreas, anterior pituitary lobe, and adrenal medulla where the relative incidence of immunoreactive cells is higher compared to syn immunoreactive cells. They have both been used for the immunohistochemical identification of NETs [10-12].

VMAT1 and 2 are acidic glycoproteins and part of a larger family of transporters termed as toxin-extruding antiporter system. Both VMATs are responsible for the uptake and storage of the monoamines dopamine, noradrenalin, adrenalin, 
and 5-hydroxytryptophane/serotonin in synaptic/secretory vesicles [2, 3]. VMAT1 is expressed in the enterochromaffin cells of the gastrointestinal tract and in the chromaffin cells of the adrenal medulla [13] while VMAT2 is found in endocrine (e.g., chromaffin cells of the adrenal medulla, enterochromaffin-like cells, ghrelin/obestatin cells, and pancreatic $\beta$ cells), as well as in non-endocrine cells (e.g., mast cells) [13-15].

In $\mathrm{NB}$, immunostaining for $\mathrm{CgA}$ and syn are usually included in the routine histopathological diagnostics. Blood concentrations of $\mathrm{CgA}$ are also frequently elevated in patients with $\mathrm{NB}$ and $\mathrm{CgA}$ can thus be used as a circulating biomarker [16-19].

The aims of this study were to investigate the immunohistochemical expression of the general NE markers $\mathrm{CgA}$, syn, SV2 and the more specific markers monoamine transporters VMAT1 and VMAT2 in NB, and to evaluate these markers' usefulness in the histopathological diagnostics. We also studied whether or not chemotherapy affects the immunoreactivity (IR).

\section{Material and Methods}

\section{Patients and Tumors}

Thirty-six tumor samples from 21 patients with NB, who were diagnosed at Uppsala University Hospital, were included. Clinical data of the patients are shown in Table 1. Two samples were excluded due to poor quality; thus, finally, 34 samples were immunostained. In 10 patients, the tumor sample before chemotherapy was obtained by surgery, in five by ultrasound-guided cutting needle biopsy. Resected tumor samples after chemotherapy were available in eight patients. In four patients, more than one tumor sample was available: One patient had two, one three, one five, and one had seven different tumor samples available. In four patients, tumor samples were available both before and after chemotherapy.

\section{Immunohistochemistry}

\section{NB Specimens}

All tissue samples were fixed in $10 \%$ buffered neutral formalin and processed to paraffin wax. Adjacent sections, approx. $4 \mu \mathrm{m}$ thick, were attached to positively charged glass slides (Superfrost@+; Menzel Gläser, Braunschweig, Germany). Hematoxylin-eosin was used as routine staining.

For immunostaining, the avidin-biotin-peroxidase complex technique (Vectastain ABC kit; Vector Laboratories Inc., Burlingame, CA) was used with diaminobenzidine as chromogen. All the sections were pretreated in a microwave oven twice for $5 \mathrm{~min}$ at $750 \mathrm{~W}$, with a Tris-buffered saline $(\mathrm{pH}$ 8.0) as retrieval solution. Endogenous peroxidase activity was quenched by incubating the sections for 20 min with $2 \%$ hydrogen peroxide in phosphate-buffered saline, $\mathrm{pH}$ 7.4.

All sections were then incubated at room temperature for 30 min with serum from the same species as the secondary antibody was raised in, before applying the primary antibody. The serum, diluted 1:5 with phosphate-buffered saline, was either normal horse serum (S-2000, Vector) or normal goat serum (S-1000 Vector).

The primary antibodies applied were mouse monoclonal vs. CgA (LK2H10, 1199021, Boehringer, Mannheim, Germany, dilution 1:16000) and vs. SV2 (NCL-SV2, 15E11, NovoCastra, Newcastle upon Tyne, UK, 1:50); rabbit polyclonal vs. syn (A0010, DakoCytomation, Glostrup, Denmark, 1:400) and VMAT2 (AB1767, Chemicon International,

Table 1 Clinical data on patients

\begin{tabular}{ll}
\hline Gender (boys/girls) & $13 / 8$ \\
Total number of specimens examined & 36 \\
Specimens obtained biopsy/surgery & $11 / 25^{*}$ \\
Specimens before/after chemotherapy & $21 / 15^{*}$ \\
Age at diagnosis (range) & 1 week-10 years and 9 months \\
Age at diagnosis (median in months) & 21 \\
INSS stage 1/2/3/4/4S & $2 / 7 / 2 / 9 / 1$ \\
Site of tumor: retroperitoneal/thoracic/neck/pelvic & $10 / 7 / 2 / 2$ \\
Survivors follow-up (range in years) & $9-24$ \\
Survivors follow-up (median in years) & 19 \\
MYCN amplification & 6 \\
Deaths & 8 \\
\hline
\end{tabular}

INSS The International Neuroblastoma Staging System, MYCN avian MYeloCytomatosis and human neuroblastoma derived homolog

*Two of the 36 specimens were excluded due to poor quality. One specimen per patient was used for semiquantitative calculation of immunoreactive cells. The specimen with the highest immunoreactivity was selected for the analysis 
Temecula, CA, 1:2400); and finally, goat polyclonal vs. VMAT1 (C-19, sc-7718, Santa Cruz Biotechnology®, Santa Cruz, CA, 1:4000). Sections were incubated with the primary antibody overnight at room temperature.

The secondary biotinylated antibodies were horse vs. mouse IgG $(\mathrm{H}+\mathrm{L})$ (BA-2000 Vector Laboratories Inc., 1:100), horse vs. goat $\operatorname{IgG}(\mathrm{H}+\mathrm{L})$ (BA-9500 Vector Laboratories Inc., 1:100), and goat vs. rabbit $\operatorname{IgG}(\mathrm{H}+\mathrm{L})$ (BA-1000) Vector Laboratories Inc., 1:100).

\section{Control Tissue}

Tissue sections from macro- and microscopically normal human gastric corpus and antral mucosa from a stomach resected due to adenocarcinoma was used as positive controls. Negative controls were generated by replacement of the primary antibody in question with non-immune serum at the same dilution with the same diluent. The non-immune sera used were mouse serum IgG1 X0931, DakoCytomation; mouse IgG1 kappa, MG1-45, Abcam®, Cambridge, UK; rabbit IgG, I-1000, Vector, and normal goat IgG, sc-2028, Santa Cruz Biotechnology ${ }^{\circledR}$. A positive control section was included in every staining batch.

A neutralization test for VMAT1 and VMAT2 was performed. The primary antiserum was incubated with the relevant antigen (VMAT1 or VMAT2 at $10 \mathrm{nmol} / \mathrm{mL}$ diluted antibody solution) at $+4 \mathrm{C}$ for $24 \mathrm{~h}$ before application to the sections. The peptides used for the neutralization test were: VMAT1, C-19, sc-7718, Santa Cruz Biotechnology ${ }^{\circledR}$ and VMAT2, AG263, Chemicon International.

\section{Semiquantitative Calculation of Immunoreactive Cells}

A semiquantitative assessment of the frequency of immunoreactive tumor cells was performed in adjacent sections by light microscopy. The frequency of the immunoreactive cells was expressed in percent intervals of the total number of tumor cells. The assessment was performed by one of the authors (LG).

\section{Results}

\section{Histopathology of Neuroblastoma Tissue Specimens}

All tumors were poorly differentiated (Schwannian stromapoor subtype). Two of the tumors were classified as ganglioneuroblastoma but only the neuroblastic cell component was analyzed. The mitotic index was high (>20 mitoses/ 10 high power fields) in all cases before chemotherapy. Necrosis was present in most tumors and calcifications were occasionally found.

\section{Expression of General NE Markers}

Seventeen of the tumors were immunostained for all three general NE markers. Thirteen of these tumor samples were stained before and five after chemotherapy. In one case (patient no. 20), immunostained sections were available both before and after chemotherapy (Table 2 and Fig. 1a-c).

\section{Before Chemotherapy}

$\mathrm{CgA}$ and SV2 were expressed in $90 \%$ or more tumors cells in nine of the 13 tumor samples, syn in five. The frequency of $\mathrm{CgA}$ and SV2 immunoreactive cells exceeded 50\% in all 13 samples, syn in eight. One case did not reveal any syn IR (Table 2).

\section{After Chemotherapy}

$\mathrm{CgA}$ and SV2 were expressed in $90 \%$ or more tumor cells in four of the five tumor samples, syn in two. The frequency of $\mathrm{CgA}$ immunorective cells exceeded $50 \%$ in all tumor samples. The corresponding frequency for SV2 and syn was observed in four and three samples, respectively (Table 2 ).

\section{Monoamine Transporters VMAT1 and 2}

Nineteen cases were immunostained for both VMAT1 and 2. Ten of the cases contained VMAT1 immunoreactive cells, 17 cases VMAT2 (Table 2, Fig. 1d, e). In two cases, immunostained sections were available both before and after chemotherapy (patients no. 13 and 20, Table 2).

\section{Before Chemotherapy}

VMAT1 IR was expressed in nine of 14 tumor samples before chemotherapy and VMAT2 in 13. Six tumors had $90 \%$ or more immunoreactive cells for VMAT2 while no tumor showed such a high IR for VMAT1 (Table 2).

\section{After Chemotherapy}

VMAT1 was expressed in two of seven tumors, VMAT2 in five (Table 2). The frequency of VMAT1 immunoreactive cells was $90 \%$ or more in one of the tumors and for VMAT2 in two (Table 2). 
Table 2 The expression of neuroendocrine markers in neuroblastoma before and after chemotherapy

\begin{tabular}{|c|c|c|c|c|c|c|c|c|c|c|}
\hline Pat. no. & Age at diagnosis & Stage & Localization & $\begin{array}{l}\text { Surgery/ } \\
\text { biopsy }\end{array}$ & Outcome & $\mathrm{CgA}$ & Syn & SV2 & VMAT1 & VMAT2 \\
\hline \multicolumn{11}{|c|}{ Before chemotherapy } \\
\hline 1 & $2 \mathrm{~m}$ & III & Thoracic & Surgery & Alive & ++++ & ++ & ++++ & 0 & +++ \\
\hline 2 & $11 \mathrm{~m}$ & II & Thoracic & Surgery & Alive & +++++ & +++++ & +++++ & ++ & +++++ \\
\hline 3 & $1 \mathrm{~m}$ & II & Thoracic & Surgery & Alive & +++++ & +++++ & +++++ & ++++ & ++++ \\
\hline 4 & $2 \mathrm{y}$ and $6 \mathrm{~m}$ & II & Thoracic & Surgery & Alive & +++++ & +++++ & +++++ & ++ & ++++ \\
\hline 5 & $8 \mathrm{mo}$ & I & Thoracic & Surgery & Alive & ++++ & + & ++++ & + & ++++ \\
\hline 6 & $1 \mathrm{y}$ and $2 \mathrm{~m}$ & II & Neck/thoracic & Surgery & Alive & +++++ & ++++ & +++++ & + & +++++ \\
\hline 7 & $2 \mathrm{~m}$ & I & Retroperitoneal & Surgery & Alive & +++++ & ++ & +++++ & ++++ & +++++ \\
\hline 9 & $2 \mathrm{y}$ and $1 \mathrm{~m}$ & II & Thoracic & Surgery & Alive & +++++ & +++++ & +++++ & ++++ & +++++ \\
\hline 10 & $1 \mathrm{w}$ & IVS & Retroperitoneal & Surgery & Alive & +++++ & 0 & +++++ & +++ & +++++ \\
\hline 11 & 7 y $8 \mathrm{~m}$ & IV & Thoracic & Surgery & Alive & +++++ & ++++ & +++++ & 0 & ++ \\
\hline 13 & $4 \mathrm{y}$ & IV & Retroperitoneal & Biopsy & Dead & Missing & Missing & +++++ & +++ & ++++ \\
\hline 15 & $7 \mathrm{~m}$ & III & Pelvic & Biopsy & Alive & +++++ & +++++ & +++++ & Missing & Missing \\
\hline 18 & $10 \mathrm{y}$ and $9 \mathrm{~m}$ & IV & Retroperitoneal & Biopsy & Dead & ++++ & ++++ & ++++ & 0 & +++++ \\
\hline 20 & $3 \mathrm{y}$ and $1 \mathrm{~m}$ & IV & Retroperitoneal & Biopsy & Dead & ++++ & ++ & ++++ & 0 & 0 \\
\hline 21 & $1 \mathrm{y}$ & IV & Retroperitoneal & Biopsy & Dead & ++ & missing & +++++ & 0 & ++++ \\
\hline \multicolumn{11}{|c|}{ After chemotherapy } \\
\hline 8 & $4 \mathrm{y}$ and $1 \mathrm{~m}$ & IV & Retroperitoneal & Surgery & Dead & +++++ & +++++ & +++++ & 0 & 0 \\
\hline 12 & $3 \mathrm{y}$ and $1 \mathrm{~m}$ & IV & Retroperitoneal & Surgery & Dead & Missing & Missing & Missing & 0 & + \\
\hline 13 & $4 \mathrm{y}$ & IV & Retroperitoneal & Surgery & Dead & Missing & +++++ & +++++ & +++++ & +++++ \\
\hline 14 & $4 \mathrm{y}$ and $8 \mathrm{~m}$ & III & Retroperitoneal & Surgery & Dead & Missing & ++++ & +++++ & 0 & +++ \\
\hline 16 & $1 \mathrm{y}$ & IV & Retroperitoneal & Surgery & Dead & +++++ & ++++ & +++++ & 0 & 0 \\
\hline 17 & $7 \mathrm{y}$ and $5 \mathrm{~m}$ & IV & Neck/thoracic & Surgery & Alive & +++++ & +++++ & +++++ & ++++ & +++++ \\
\hline 19 & $1 \mathrm{y}$ and $10 \mathrm{~m}$ & II & Pelvic & Surgery & Alive & +++ & + & ++ & Missing & Missing \\
\hline 20 & $3 \mathrm{y}$ and $1 \mathrm{~m}$ & IV & Retroperitoneal & Surgery & Dead & +++++ & ++ & +++++ & 0 & ++ \\
\hline
\end{tabular}

The frequency of immunoreactive cells is expressed in percent intervals of the total number of tumor cells. If two or more sections were assessed from the same case at the same location, the highest value is presented. In one case, tissue samples were available both before and after chemotherapy ,$+++++ \geq 90 \% ;++++, 70<90 \% ;+++, 50<70 \% ;++, 10<50 \% ;+,<10 \% ; 0$, no reactive cells

CgA chromogranin A, syn synaptophysin, SV2 synaptic vesicle protein 2, VMAT1 vesicular monoamine transporter 1, VMAT 2 vesicular monoamine transporter 2, $y$ year(s), $m$ months, $w$ week

\section{Comparison of the Immunoreactivity Between All Studied Neuroendocrine Markers}

Immunostained sections with the general markers and the monoamine transporters were available in 15 cases, 12 before and four after chemotherapy. In one case, immunostained sections were available both before and after chemotherapy. $\mathrm{CgA}$ and SV2 were more frequently expressed in the samples than syn. VMAT1 was less expressed than the other markers. Syn and VMAT2 showed a similar staining frequency (Table 3).

\section{Controls}

Immunoreactive cells corresponding to the three general markers and the monoamine transporters were represented in the gastric mucosa. More CgA, syn, and SV2 immunoreactive cells were seen than VMAT1 and 2 immunoreactive cells.
Negative controls exhibited no IR. All immunostaining was cytoplasmic.

\section{Discussion}

We have shown that IR for SV2 is a useful marker for identifying NE differentiation in NB, a property it shares with CgA. Syn was somewhat inferior to identify NE tumor cells compared to the other two general markers. In a previous study of NB, syn was suggested to be a better NE marker than $\mathrm{CgA}$ [16]. The difference between our and their study may depend on tissue processing and choice of antibodies. They used frozen sections and a monoclonal antibody while we used formalin-fixed, deparaffinized sections and a polyclonal antibody. 
Fig. 1 Immunohistochemical demonstration of $\mathbf{a}$;

chromogranin $\mathbf{a}, \mathbf{b}$;

synaptophysin, $\mathbf{c} ; \mathrm{SV} 2, \mathbf{d}$;

VMAT1, e; VMAT2 in a

neuroblastoma (pat. no. 4).

Almost all tumor cells display

strong immunoreactivity to the

general neuroendocrine markers

and VMAT2. Few tumor cells

show VMAT1 immunoreactivity.

The bar in picture $\mathrm{A}$ is $50 \mu \mathrm{m}$
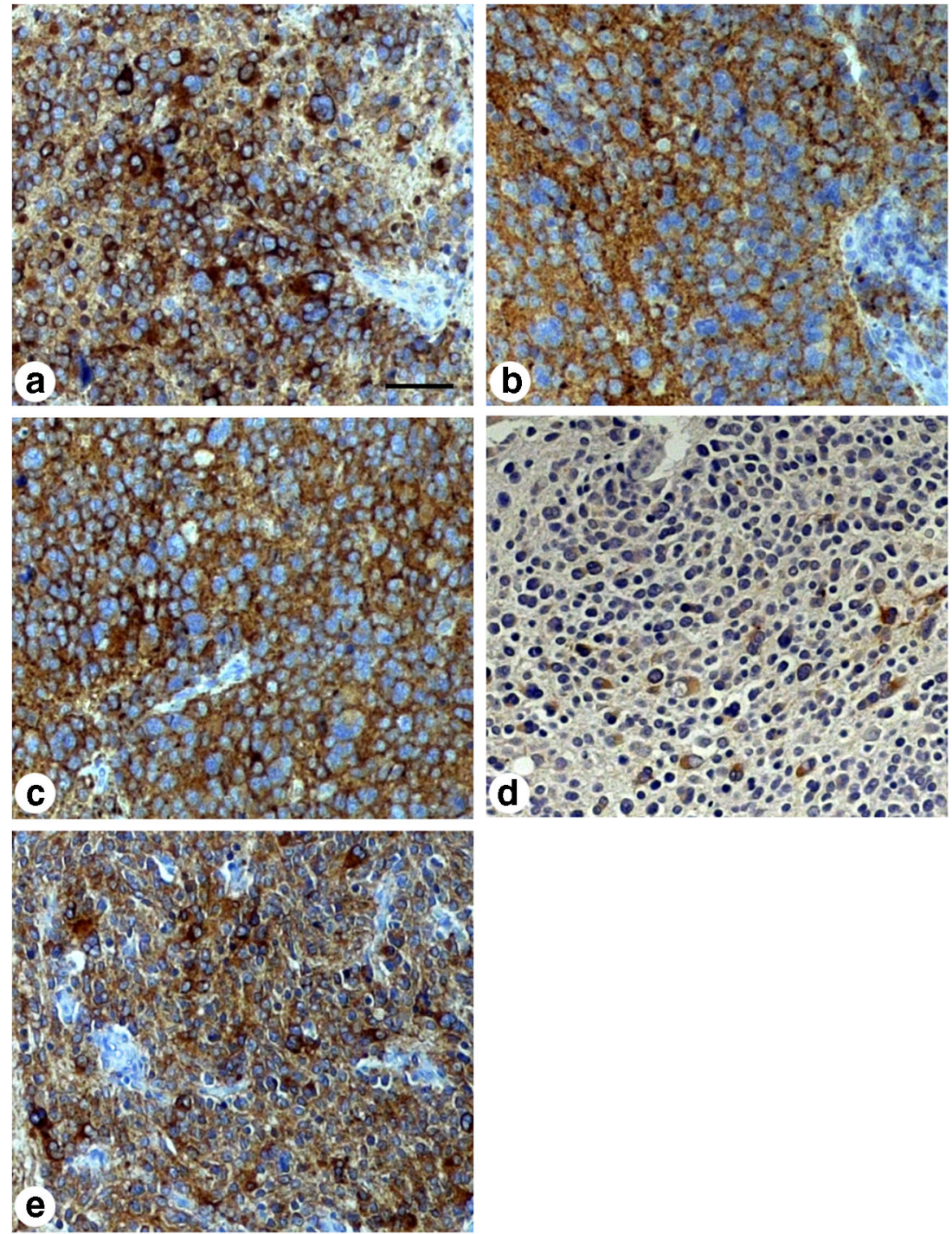

SV2 has earlier been reported to identify most NE cell types and NE tumors. It is thus an important NE marker, comparable to $\mathrm{CgA}[10,11]$. Portela-Gomes et al. showed that SV2 identified most NE cell and tumor types in different organs, a property it shared with $\mathrm{CgA}$ and syn. There are even NETs (e.g., rectal NETs, L-cell type) where SV2 is more reliable diagnostically than both $\mathrm{CgA}$ and syn. Similar staining results were reported by Jakobsen et al. who studied the expression of $\mathrm{Sv} 2$ and $\mathrm{CgA}$ in endocrine tumors of the gastrointestinal tract and pancreas. However, none of these studies included NB.

We also studied whether or not chemotherapy influenced the marker IR. Our findings showed that chemotherapy had no obvious such effect, possibly with the exception of VMAT1. This latter marker showed a larger proportion of immunoreactive cells before than after chemotherapy. There were, however, too few cases to make any certain conclusions.
In a previous study of 76 children with high risk, metastatic $\mathrm{NB}$, the expression of VMAT1 and VMAT2 was analyzed and the number of tumor cases expressing VMAT2 exceeded that of VMAT1 (75\% vs. 62\%) [20]. We found similar result to theirs, $84 \%$ vs. $57 \%$, respectively. Our study included patients of all stages with or without metastases.

A limitation of the present study is the scant number of cases included due to the paucity of the disease. Furthermore, not all the cases were immunostained for markers in question because there was no more available material at the pathology department.

We conclude that SV2 is a useful general NE marker for $\mathrm{NB}$, similar to CgA. Syn was somewhat inferior as general marker for NB compared to the other two. Tissue processing and choice of antibody may contribute to this difference. VMAT1 and VMAT2 were expressed in several NB; more NB expressed VMAT2 than VMAT1. Also in the individual cases, the fraction of VMAT2 immunoreactive cells usually 
Table 3 Frequency of immunoreactive tumor cells in cases immunostained with the complete sequence of neuroendocrine markers

\begin{tabular}{llllll}
\hline$\geq 90 \%$ & $70-$ & $50-$ & $10-$ & $<10 \%$ & $0 \%$ \\
+++++ & $<90 \%$ & $<70 \%$ & $<50 \%$ & + & 0 \\
& ++++ & +++ & ++ & & \\
\hline
\end{tabular}

Before chemotherapy $n=12$

\begin{tabular}{|c|c|c|c|c|c|}
\hline $\mathrm{CgA}$ & 8 & 4 & 0 & 0 & 0 \\
\hline syn & 4 & 3 & 0 & 3 & 1 \\
\hline SV2 & 8 & 4 & 0 & 0 & 0 \\
\hline VMAT1 & 0 & 3 & 1 & 2 & 2 \\
\hline VMAT2 & 6 & 4 & 1 & 1 & 0 \\
\hline \multicolumn{6}{|c|}{ After chemotherapy $n=4$} \\
\hline $\mathrm{CgA}$ & 4 & 0 & 0 & 0 & 0 \\
\hline SYN & 2 & 1 & 0 & 1 & 0 \\
\hline SV2 & 4 & 0 & 0 & 0 & 0 \\
\hline VMAT1 & 0 & 1 & 0 & 0 & 0 \\
\hline VMAT2 & 1 & 0 & 0 & 1 & 0 \\
\hline
\end{tabular}

Data are extracted from Table 2 but simplified for comparison between markers. If two or more sections were assessed from the same case at the same location, the highest value is presented. In one case, tissue samples were stained both before and after the treatment

CgA chromogranin A, syn synaptophysin, SV2 synaptic vesicle protein 2, VMAT1 vesicular monoamine transporter 1, VMAT 2 vesicular monoamine transporter 2

exceeded that of VMAT1. VMAT2 was similar to syn as a NE marker. Chemotherapy did not influence the IR in an obvious way. Since our cohort was small, our findings should be confirmed in larger, prospective studies. Furthermore, our results could be compared to other NE markers expressed in NB.

Acknowledgments We would like to express our sincere gratitude to Erik Lampa, Ph.D., Uppsala Clinical Research Center, for the statistical advice and Malin Grönberg, Ph.D., Department of Medical Sciences, Endocrine Oncology, for the technical support, both at Uppsala University, Uppsala, Sweden.

Funding Information Open access funding provided by Uppsala University. This work was sponsored with funds from the Lions foundation for cancer research at Uppsala University Hospital and the Swedish Childhood Cancer Foundation.

\section{Compliance with Ethical Standards}

Ethical Approval The research protocol was reviewed and approved by the Regional Ethical Review Board in Uppsala (ref. number 2006/361). Informed consent was obtained for all included patients.

Open Access This article is distributed under the terms of the Creative Commons Attribution 4.0 International License (http:// creativecommons.org/licenses/by/4.0/), which permits unrestricted use, distribution, and reproduction in any medium, provided you give appropriate credit to the original author(s) and the source, provide a link to the Creative Commons license, and indicate if changes were made.

\section{References}

1. Machado JD, Diaz-Vera J, Dominguez N, Alvarez CM, Pardo MR, Borges R (2010) Chromogranins A and B as regulators of vesicle cargo and exocytosis. Cell Mol Neurobiol 30 (8):1181-1187. doi: https://doi.org/10.1007/s10571-010-9584-y

2. Schafer MK, Weihe E, Eiden LE (2013) Localization and expression of VMAT2 aross mammalian species: a translational guide for its visualization and targeting in health and disease. Adv Pharmacol 68:319-334. doi:https://doi.org/10.1016/B978-0-12-411512-5. 00015-4

3. Wimalasena K (2011) Vesicular monoamine transporters: structurefunction, pharmacology, and medicinal chemistry. Med Res Rev 31 (4):483-519. doi:https://doi.org/10.1002/med.20187

4. Mendoza-Torreblanca JG, Vanoye-Carlo A, Phillips-Farfan BV, Carmona-Aparicio L, Gomez-Lira G (2013) Synaptic vesicle protein 2A: basic facts and role in synaptic function. Eur J Neurosci 38 (11):3529-3539. doi:https://doi.org/10.1111/ejn.12360

5. Valtorta F, Pennuto M, Bonanomi D, Benfenati F (2004) Synaptophysin: leading actor or walk-on role in synaptic vesicle exocytosis? Bioessays 26 (4):445-453. doi:https://doi.org/10.1002/ bies. 20012

6. O'Connor DT, Burton D, Deftos LJ (1983) Immunoreactive human chromogranin A in diverse polypeptide hormone producing human tumors and normal endocrine tissues. J Clin Endocrinol Metab 57 (5):1084-1086. doi:https://doi.org/10.1210/jcem-57-5-1084

7. Rosenbaum JN, Guo Z, Baus RM, Werner H, Rehrauer WM, Lloyd RV (2015) INSM1: A Novel Immunohistochemical and Molecular Marker for Neuroendocrine and Neuroepithelial Neoplasms. Am J Clin Pathol 144 (4):579-591. doi:https://doi.org/10.1309/ AJCPGZWXXBSNL4VD

8. Wang H, Krishnan C, Charville GW (2019) INSM1 Expression in Peripheral Neuroblastic Tumors and Other Embryonal Neoplasms. Pediatr Dev Pathol 1093526619843725. doi:https://doi.org/10. $1177 / 1093526619843725$

9. Li YC, Kavalali ET (2017) Synaptic Vesicle-Recycling Machinery Components as Potential Therapeutic Targets. Pharmacol Rev 69 (2):141-160. doi:https://doi.org/10.1124/pr.116.013342

10. Portela-Gomes GM, Lukinius A, Grimelius L (2000) Synaptic vesicle protein 2, A new neuroendocrine cell marker. Am J Pathol 157 (4):1299-1309. doi:https://doi.org/10.1016/S0002-9440(10) 64645-7

11. Jakobsen AM, Ahlman H, Wangberg B, Kolby L, Bengtsson M, Nilsson O (2002) Expression of synaptic vesicle protein 2 (SV2) in neuroendocrine tumours of the gastrointestinal tract and pancreas. $\mathrm{J}$ Pathol 196 (1):44-50. doi:https://doi.org/10.1002/path.1002

12. Wiedenmann B, Franke WW, Kuhn C, Moll R, Gould VE (1986) Synaptophysin: a marker protein for neuroendocrine cells and neoplasms. Proc Natl Acad Sci U S A 83 (10):3500-3504

13. Erickson JD, Schafer MK, Bonner TI, Eiden LE, Weihe E (1996) Distinct pharmacological properties and distribution in neurons and endocrine cells of two isoforms of the human vesicular monoamine transporter. Proc Natl Acad Sci U S A 93 (10):5166-5171

14. Anlauf M, Eissele R, Schafer MK, Eiden LE, Arnold R, Pauser U, Kloppel G, Weihe E (2003) Expression of the two isoforms of the vesicular monoamine transporter (VMAT1 and VMAT2) in the endocrine pancreas and pancreatic endocrine tumors. J Histochem Cytochem 51 (8):1027-1040. doi:https://doi.org/10.1177/ 002215540305100806

15. Tsolakis AV, Grimelius L, Stridsberg M, Falkmer SE, Waldum HL, Saras J, Janson ET (2009) Obestatin/ghrelin cells in normal mucosa and endocrine tumours of the stomach. Eur J Endocrinol 160 (6): 941-949. doi:https://doi.org/10.1530/EJE-09-0001

16. Molenaar WM, Baker DL, Pleasure D, Lee VM, Trojanowski JQ (1990) The neuroendocrine and neural profiles of neuroblastomas, 
ganglioneuroblastomas, and ganglioneuromas. Am J Pathol 136 (2):375-382

17. Georgantzi K, Skoldenberg EG, Stridsberg M, Kogner P, Jakobson A, Janson ET, Christofferson RHB (2018) Chromogranin A and neuron-specific enolase in neuroblastoma: Correlation to stage and prognostic factors. Pediatr Hematol Oncol:1-10. doi:https:// doi.org/10.1080/08880018.2018.1464087, 35

18. Carvalho Ade C, Parra ER, Zerbini MC, Alves VA, Capelozzi VL, Antonangelo L (2007) Morphometric evaluation of NB84, synaptophysin and AgNOR is useful for the histological diagnosis and prognosis in peripheral neuroblastic tumors (pNTs). Clinics (Sao Paulo) 62 (6):731-740

19. Hsiao RJ, Seeger RC, Yu AL, O'Connor DT (1990) Chromogranin $\mathrm{A}$ in children with neuroblastoma. Serum concentration parallels disease stage and predicts survival. J Clin Invest 85 (5):15551559. doi:https://doi.org/10.1172/JCI114604
20. Temple W, Mendelsohn L, Kim GE, Nekritz E, Gustafson WC, Lin L, Giacomini K, Naranjo A, Van Ryn C, Yanik GA, Kreissman SG, Hogarty M, Matthay KK, DuBois SG (2016) Vesicular monoamine transporter protein expression correlates with clinical features, tumor biology, and MIBG avidity in neuroblastoma: a report from the Children's Oncology Group. Eur J Nucl Med Mol Imaging 43 (3): 474-481. doi:https://doi.org/10.1007/s00259-015-3179-2

Publisher's Note Springer Nature remains neutral with regard to jurisdictional claims in published maps and institutional affiliations. 